\title{
COMPUTER AIDED INNOVATON OF CRANKSHAFTS USING GENETIC ALGORITHMS
}

\author{
Humberto Aguayo Téllez, Noel León Rovira ${ }^{2}$ \\ ${ }^{\prime}$ Center for Innovation in Design \& Technology (CIDT), ITESM; Email: haguayo@itesm.mx. \\ ${ }^{2}$ Professor CIDT, Director Research Program Creativity and Innovation in Engineering, \\ ITESM, Campus Monterrey, Chairperson WG5.4 Computer Aided Innovation IFIP T;. Email: \\ noel.leon@itesm.mx. Phone: +528181582012 .
}

\begin{abstract}
In this paper a method is described for determining the design unbalance of crankshafts and also the recommended procedure for a balanced design strategy. The use of a search tool for solutions is suggested based on Genetic Algorithms (GA). GAs have been used in different applications, one of them is the optimization of geometric shapes, a relatively recent area with high research potential. The interest towards this field is growing, and it is anticipated that in the future mechanical engineering will be an area where many applications of shape optimization will be widely applied.
\end{abstract}

Key words: Genetic Algorithms, Splines, Unbalance

\section{INTRODUCTION}

The objective of the balance analysis of engine crankshafts is to develop its geometry so that a specified previous unbalance is obtained on two correction planes. These two planes lie where the outermost counterbalances are located. This previous unbalance represent a value of mass by radial position which effect will be eliminated by means of drilled holes around such counterbalances (at the correction planes, for instance) to values near zero, taking in count the effect of pistons and rods weights (these last commonly replaced for equivalent "fictitious" weights). This intentional previous unbalance is designed just for the drilling to take out mass

Please use the following format when citing this chapter:

Téllez, Humberto Aguayo, Rovira, Noel León, 2006, in International Federation for Information Processing (IFIP), Volume 207, Knowledge Enterprise: Intelligent Strategies In Product Design, Manufacturing, and Management, eds. K. Wang, Kovacs G., Wozny M., Fang M., (Boston: Springer), pp. 471-476. 
(unbalance mass) from the external profile surface of the counterweight, at a limited zone, restricted because of the engine geometry and manufacturing process. Figure 1 shows a schematic sketch of a crankshaft.

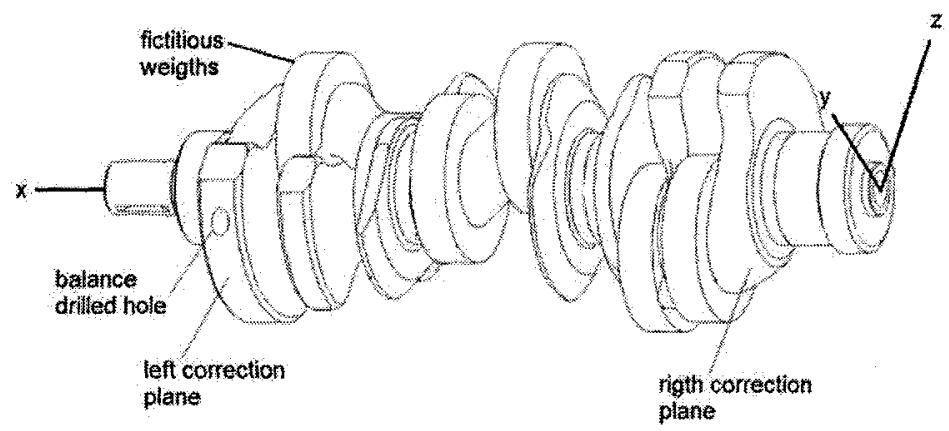

Figure 1. Combustion Engine Crankshaft

In the pivoted crankshaft turning with constant angular velocity the sum of moments produced by the differentials of mass on its location is equal to a bending moment acting on the crankshaft. The equations that govern the dynamic behavior of rotating crankshafts are:

$$
\begin{aligned}
& \Sigma \mathrm{M}_{\mathrm{x}}=0 \\
& \Sigma \mathrm{M}_{\mathrm{y}}=\mathrm{I}_{\mathrm{xz}} \omega^{\wedge} 2 \\
& \Sigma \mathrm{M}_{\mathrm{z}}=-\mathrm{I}_{\mathrm{xy}} \omega^{\wedge} 2 \\
& \text { Restricted to } \\
& \Sigma \mathrm{F}=0 ;
\end{aligned}
$$

The sum of the inertia forces produced by each differential of the crankshaft mass and the mass from the pistons and rods is equal to the reactions on the supports at the rotation fixations [1]. Those equations allow finding the equivalent unbalance. The procedure for doing so is described next.

The sum of moments of the equation is equal to the resultant of the moment caused by the centrifugal force due to the crankshaft mass at the center of gravity, and the moment caused by the unbalance masses at the correction planes. It is a common practice to take this sum of moments about the left correction plane (into the counterweight close to the flange) in order to eliminate the moment caused by the left unbalance mass and finding the unbalance on the right plane: 


$$
\begin{aligned}
& \mathrm{m}_{\mathrm{R}} \mathrm{r}_{\mathrm{Ry}}=\left((l / 2) \mathrm{m}_{\mathrm{g}} \mathrm{r}_{\mathrm{gy}}+\mathrm{I}_{\mathrm{xy}}\right) / l \\
& \mathrm{~m}_{\mathrm{R}} \mathrm{r}_{\mathrm{Rz}}=\left((l / 2) \mathrm{m}_{\mathrm{g}} \mathrm{r}_{\mathrm{gz}}+\mathrm{I}_{\mathrm{xz}}\right) / l
\end{aligned}
$$

where $l$ is the distance between the two correction planes, $m_{\mathrm{g}}$ is the mass of the crankshaft, $r_{\mathrm{gy}}$ and $\mathrm{r}_{\mathrm{gz}}$ are the coordinates $\mathrm{y}$ and $\mathrm{z}$ of the center of gravity. The same way moments are taken about the right correction plane to find the unbalance on the left plane:

$$
\begin{aligned}
& \mathrm{m}_{\mathrm{L}} \mathrm{r}_{\mathrm{Ly}}=\left((l / 2) \mathrm{m}_{\mathrm{g}} \mathrm{r}_{\mathrm{gy}}-\mathrm{I}_{\mathrm{xy}}\right) / l \\
& \mathrm{~m}_{\mathrm{L}} \mathrm{r}_{\mathrm{Lz}}=\left((l / 2) \mathrm{m}_{\mathrm{g}} \mathrm{r}_{\mathrm{gz}}-\mathrm{I}_{\mathrm{xz}}\right) / l
\end{aligned}
$$

The mass properties of interest $\left(\mathrm{m}_{\mathrm{g}}, \mathrm{r}_{\mathrm{gy}}, \mathrm{r}_{\mathrm{gz}}\right.$ and the inertia products $\mathrm{I}_{\mathrm{xy}}$ and $I_{x z}$ ) of the crankshaft model can be obtained from the CAD software, orienting the geometry respect to a system of coordinates with its $\mathrm{x}$ axis towards the flange end upon the rotation axis and the $\mathrm{z}$ axis in the proper vertical orientation, using the proper info commands of the commercial CAD software. It is worth noticing that these equations do not depend on the angular velocity of the crankshaft.

If it is specified a target for unbalance $\left(m_{L} r_{L} y m_{R} r_{R}\right)$ the difference between the target and the current unbalance is a set of vectors that is going to be minimized by making modifications to the crankshaft geometry.

These vectors are dependent of the counterweight shape. The search for an optimal shape can be extensively time consuming but different strategies can be used to reduce the search time and effort for obtaining an optimal geometry which consider the constrains of the most important parameters involved in the crankshaft performance and manufacturing process such as weight and the filling of cavities during the forging process.

\section{STRATEGY TO OPTIMIZE THE UNBALANCE}

Next the general method is described for developing a balance optimization procedure using Genetic Algorithms (GA) [2]. GAs have been used in different applications [3], one of them is the optimization of geometric shapes, a relatively recent area with high research potential. The first step is to have a 3D CAD parametric model of the crankshaft. From this model the mass properties required to calculate the unbalance are obtained (using the equations shown earlier).

Now that the difference between the unbalance target and the CAD model unbalance is known. The next step is to modify the CAD geometry for minimizing this difference. This implies two restrictions:

- the weight of the crankshaft should not be increased 
- the filling process of die cavities during forging should remain unaffected or even improved.

Because unbalance depends on location and amount of mass, it is not straightforward to modify the counterweights. On the other side, only certain locations of the counterweights are allowed to be modified to adjust balance.

It was decided to substitute with splines the profile of the counterweights close to the initial design. This process has been called "splinization". A procedure that was developed at the Center for Innovation on Design and Technology has been used for this substitution [4]. From this initial with splines adjusted profiles, variations are made by changing the positions of the control points. Figure 2 shows the "splinization" strategy to modify the profile of a counterweight for one of the possible profiles represented.

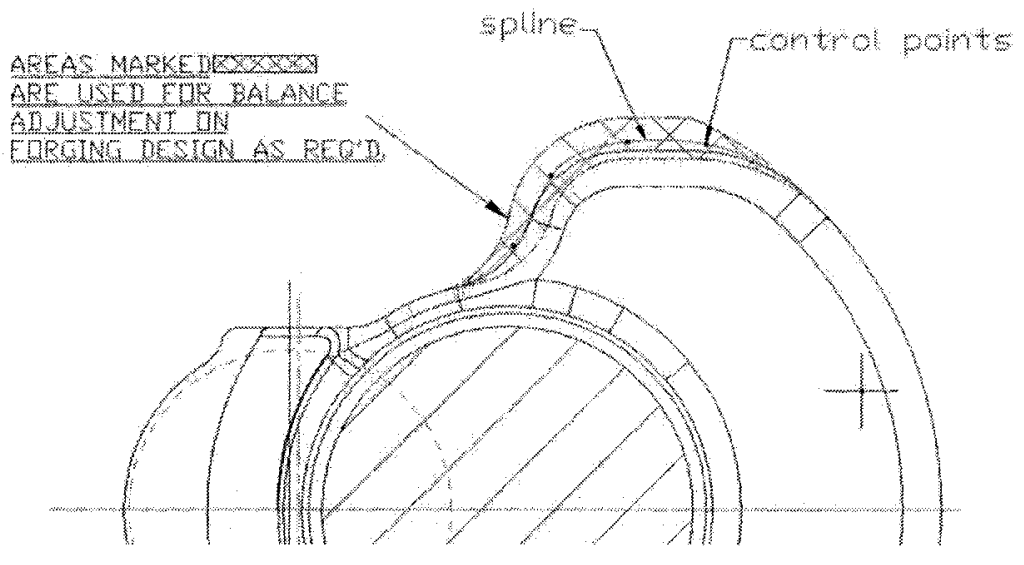

Figure2. Profile of counterweight represented by a spline

The two main reasons for using splines instead of the traditional arcs are:

1. arcs contain fewer degrees of freedom for manipulating geometry (just the center, radius and angle) and

2. the second derivative of a set of arcs contains sharp edges, something not so good for material fluency in the forging dies.

The splinization of the counterweights needs a codification of the control points to form the chromosomes of the Genetic Algorithm. This codification was achieved as follows: From each control point only the $Y$ coordinate will be used as the $\mathrm{X}$ coordinate may remain fixed in proper locations. For each $Y$ coordinate an upper and a lower value are defined, those limits are then codified as +1 and -1 and these define the phenotype. Table 1 shows these 
first three values with the upper and lower limits and its codification as $+1,0$ and -1 .

Table 1. First four $\mathrm{Y}$ coordinates of the CW1 spline

\begin{tabular}{|c|l|l|l|l|l|l|l|l|}
\hline & \multicolumn{3}{|c|}{ CW1 TOP } & \multicolumn{4}{c|}{ CW1 TOP CODIFIED } \\
\hline upper & 51 & 66 & 73 & 78 & 1 & 1 & 1 & 1 \\
\hline & 48.33 & 62.47 & 71.21 & 76.2 & 0 & 0 & 0 & 0 \\
\hline lower & 46 & 60 & 68 & 74 & -1 & -1 & -1 & -1 \\
\hline
\end{tabular}

Because splines with at least four free control points are used, i.e. on top and bottom side profiles of counterweights number $1,2,8 \& 9$ the chromosome must have at least $4 \times 2 \times 4=32$ "genes". The genetic algorithm is going to be run with an initial population representing different counterweight shapes for a crankshaft.

Each resulting crankshaft unbalance is evaluated according to the equations derived before and those that have the smallest difference vectors are combined to form a new population until the unbalance target within a certain tolerance is reached. Fig 3 . Shows the proposed algorithm.

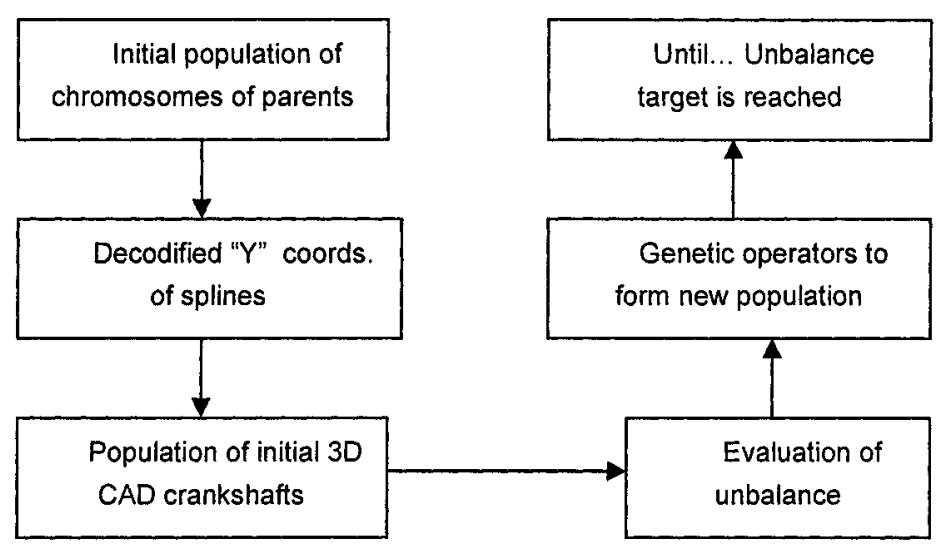

Figure 3. Proposed algorithms for balance optimization using Genetic operators

The use of commercial software I-SIGTH ${ }^{\mathrm{TM}}$ is required to integrate the genetic algorithms programmed in MATLAB ${ }^{\mathrm{TM}}$ with the modeling of CAD crankshafts in PRO/ENGINEER ${ }^{\mathrm{TM}}$

\section{CONCLUSIONS}


It is possible to optimize the balance on forging crankshafts in order to take the performance of the combustion engine far beyond the limits reached by conventional methods, using strategies of evolutionary computation. The use of Genetic Algoritms on crankshaft balance is based on the premise that the geometry of counterweights can be modified automatically by algorithms that optimize critical parameters of the engine performance

Further work is required to find the best approach on taking the new counterweights profiles under the filling of die cavities because it is perceived that these new profiles are better than conventional rounded profiles

As sketches are controlled through its parametric structure using control points through the splinization approach, a solution is presented for substituting the initial parametric structure by a new size controlling structure that allows parametric similar control of the size of parts and features being analyzed as the genetic algorithms provide.

On the other side, as parameters are used as dimensioning basis for drawings further analysis is required on how to achieve a dimensioning structure (design for manufacturing) of parts that have been "splinized".

\section{REFERENCES}

1. Rao. Mechanical Vibrations. Wesley

2. Goldberg DE. Genetic algorithms in search, optimization and machine learning. Reading, MA: Addison-Wesley; 1989. ISBN 0-201-15767-5.

3. J. Lampinen. Cam shape optimization by genetic algorithm. Computer Aided Design 35 (2003) $727-737$.

4. Noel Leon, José Maria Cueva, Jorge Gutiérrez, David Silva. Automatic Shape Variations in 3D CAD Environments. 1ST IFIP-TC5 Working Conference on Computer Aided Innovation. November 14-15 2005, Ulm, Germany 